\title{
A Deterministic Analysis of the Effectiveness of Non-Clinical Approaches in the Control of Transimission of Schistosomiasis: Case Study of Mwea Irrigation Scheme, Kenya
}

\author{
Jane N. Murungi, Stephen Karanja, Paul Wanjau
}

\begin{abstract}
Schistosomiasis commonly known as bilharzia is regarded by W.H.O as a neglected tropical disease. It affects the intestines and the urinary system preferentially, but can harm other systems in the body. The disease is a health concern among majority of the population in Mwea irrigation scheme in Kenya and indeed other tropical countries. This paper documents a deterministic analysis of the effectiveness of non-clinical approaches in the control of transmission of schistosomiasis in the region. A SIR based mathematical model that incorporates media campaigns as a control strategy of reducing transmission of the disease is used. The model considers behavior patterns of hosts as the main process of transmission of the disease. The dynamics of these processes is expressed in terms of ordinary differential equations deduced from the human behavior patterns that contribute to the spread of the disease. The reproduction number $R_{0}$ and equilibrium points both DFE and EE are obtained. The stabilities of these equilibrium points are analyzed in reference to the reproduction number $\left(\mathbf{R}_{0}\right)$. Secondary data is used in the mathematical model developed and in the prediction of the dynamics estimated in the model for a period of five years. Numerical simulation was carried out and results represented graphically. The results of the simulation show that the infection decreased from 75108 to about 35000 and the susceptible from 325142 to 50000 respectively in a period of five years. From the analysis, the DFE point is asymptotically stable when $R$ _ $0<1$.Sensitivity analysis of parameters was carried out using partial differentiation. The results show that the sensitivity index of most parameters are inversely proportional to $\mathbf{R}_{\mathbf{0}}$ which will reduce schistosomiasis infection. From the results, incorporation of media campaigns as a control strategy significantly reduces transmission of the disease. The results will be useful to MOH to enhance media campaigns to prevent spread of schistosomiasis in Mwea Irrigation scheme and other endemic areas.
\end{abstract}

Keywords - Disease free equilibrium and endemic equilibrium, local and global stabilities of equilibrium points, reproduction number, Schistosomiasis, sensitivity analysis.

\section{INTRODUCTION}

Human Schistosomiasis is the third most devastating tropical diseases in the world after malaria and intestinal helminthiasis according to the World Health Organization [37]. Schistosomiasis also referred to as Bilharzia or snail fever. Human schistosomiasis is a parasitic disease caused by parasitic worms that infects multiple organs including the liver, intestine, bladder and urethra. Clinical manifestations of schistosomiasis include fever and headache. Infection can occur in several sites including bile ducts, intestine and bladder. The disease may be eliminated with praziquantel as it is currently the only effective treatment of the disease; vaccines and gene therapy are proposed as future treatment plans. kato-katz method and PCR are detection tools for people with schistosomiasis.

Environmental and climatic factors play an important role in the geographical distribution of the disease. In Kenya, Schistosomiasis is mainly prevalent around Lake Victoria, Kwale county and Mwea irrigation scheme located in Kirinyaga county. The main socio-economic activity in this area is rice growing through irrigation using water from river Thiba and Nyamindi. Farmers spend most of their time in the field during the day and there are no toilet facilities in the vast open area in the paddies therefore water canals are the most likely places for defecation [21].

A study conducted in all primary schools in Mwea Division by Eastern and Southern Africa Center for International Parasite Control (ESACIPAC), indicated an overall prevalence of $36 \%$ in Schistosoma mansoni with more than $50 \%$ of schools within the irrigation area demonstrating infection rate of $50 \%$ and above in S. mansoni infection. School-age children therefore form a high risk group for soil transmitted helminths infections and schistosomiasis. Movements of people from rural to urban areas can cause the spread of schistosomiasis, seasonal migration of employees and refugees can also lead to outbreaks of the disease [23].

Published on December 14, 2021.

J. N. Murungi, Department of Mathematics, Meru University of Science and Technology, Meru, Kenya.

(e-mail: janenkirotemurithi@gmail.com)

S. Karanja, Department of Mathematics, Meru University of Science and Technology, Meru Kenya.

(e-mail: skaranja@must.ac.ke)

P. Wanjau, School of Mathematics and Actuarial Science, The Technical University of Kenya, Nairobi, Kenya.

(e-mail: mainapaul14@gmail.com) 


\section{MODEl ANALYSiS}

\section{A. Model Description}

In our model the total population is divided into five compartments depending on the epidemiological status of individuals; these include susceptible $\mathrm{S}(\mathrm{t})$, symptomatically infected I $(\mathrm{t})$, recovered R $(\mathrm{t})$, susceptible exposed to media $S_{m}(\mathrm{t})$ and have avoided infection, level of snail concentration in contaminated water $\mathrm{B}(\mathrm{t})$. The susceptible population increases due to the incoming of individuals who come into contact with contaminated water at the rate of $\Lambda_{1}$ and recovered individuals who join susceptible class at the rate of $\tau$. The parameters and their descriptions are given in Table II. The assumptions of the model include, the total population of individuals is not constant, controls are implemented continuously, treatment is introduced to the infected population, awareness leads to the reduction in new infections and re-infections, individuals in each compartment are homogenously mixing with each other and on recovery there is temporary immunity. Fig. 1 represent flow of the model where arrows represent movement of individuals from one compartment to the next and dotted arrows show the effect of media campaigns.

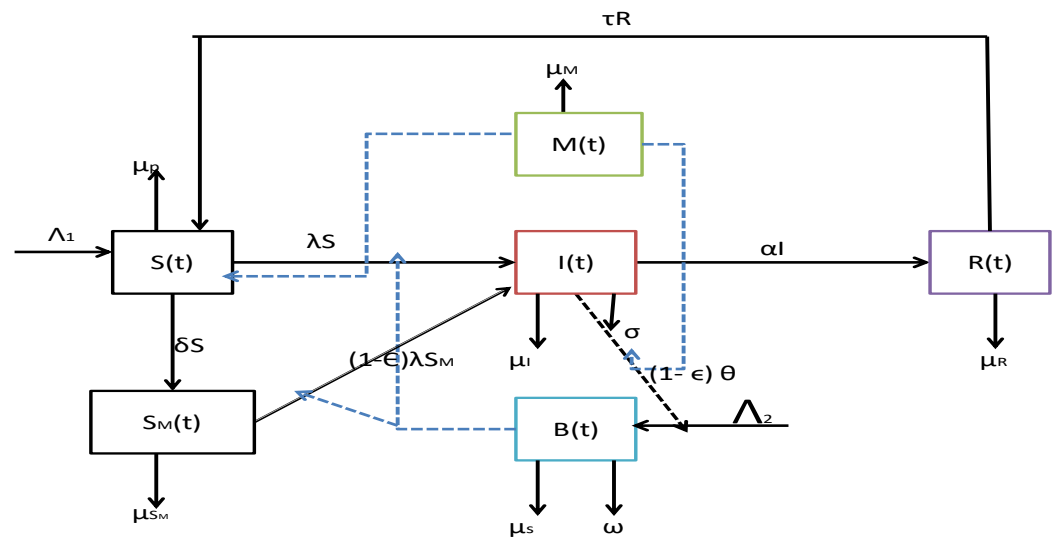

Fig. 1. Schematic flow chart of Schistosomiasis model.

Model equations from the flow chart

$$
\left\{\begin{array}{c}
\frac{d s}{d t}=\Lambda_{1}+\tau \mathrm{R}-\left(\mu_{\mathrm{p}}+\lambda_{1}\right) \mathrm{S}-\delta \mathrm{S} \\
\frac{d I}{d t}=\lambda S+(1-\epsilon) \lambda_{1} S_{M}-\left(\alpha+\mu_{I}+\sigma\right) I \\
\frac{d R}{d t}=\alpha I-\left(\mu_{R}+\tau\right) R \\
\frac{d S_{M}}{d t}=\delta S-\mu_{S_{M}} S_{M}-(1-\epsilon) \lambda_{1} S_{M} \\
\frac{d B}{d t}=\Lambda_{2}+(1-\epsilon) \theta I-\left(\mu_{S}+\omega\right) B
\end{array}\right.
$$

\section{B. Boundedness}

The model monitors dynamics of a disease involving human population hence it is important to show the limits within which the population lies. The carrying capacity in this case is the total of the equations with human population $\mathrm{N}$.

$\frac{d N}{d t}=\Lambda_{1}-\left(\mu_{p} S+\mu_{I} I+\mu_{R} R+\mu_{S_{M}} S_{M}\right.$

Taking the derivatives of total population along the solution path we obtain $\frac{d N}{d t}+\mu N \geq \Lambda_{1}$ After integrating (2) we obtain:

$N \geq \frac{\Lambda_{1}}{\mu}+K e^{-\mu t}$

where $\mathrm{K}$ is a constant of integration

$\operatorname{But}\left(S+I+R+S_{M)}\right)$ is $\mathrm{N}$ then When $\mathrm{t}=0\left(S+I+R+S_{M}\right) \geq K$ Where $\mathrm{K}$ is a constant hence $N \geq K$.

\section{Positivity}

Positivity is obtained by letting $S(t), I(t), R(t), B(t)$ to be any solutions of a system of equations with non-negative initial conditions $S(0) \geq 0, I(0) \geq 0, R(0) \geq 0, B(0) \geq 0$. We solve (1) we obtain

$\frac{d s}{d t} \geq-\left(\mu_{p}+\lambda+\delta\right) S$ 
Integrating equation (4) by separation of variables we obtain $\frac{d s}{\mathrm{~s}} \geq-\left(\mu_{\mathrm{p}}+\lambda+\delta\right) \mathrm{dt}, S(t) \geq H e^{-\left(\mu_{\mathrm{p}}+\lambda+\delta\right) \mathrm{t}}$. Where $\mathrm{H}$ is a constant. When $t=0, S(0) \geq H e^{-\left(\mu_{\mathrm{p}}+\lambda+\delta\right) 0} \geq 0$ (hence positive). We solve the other equations.

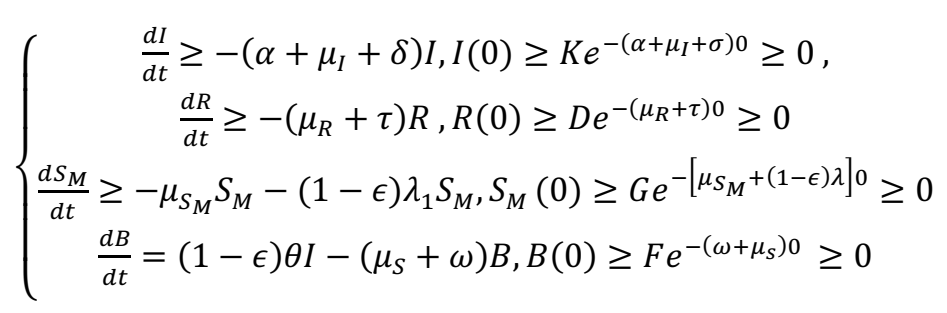

(5) above shows that system 1 remains positive at all time $t \geq 0$.

D. Basic Reproduction Number and Disease Free Equilibrium Point

Disease free equilibrium is obtained by setting model system of equations 1 as $\frac{d S}{d t}=\frac{d I}{d t}=\frac{d R}{d t}=\frac{d S_{M}}{d t}=\frac{d B}{d t}=0$ and in the absence of the disease $I=B=R=0$ hence the system of (1) reduces to (6)

$\left\{\begin{array}{c}\Lambda_{1}-\left(\mu_{\mathrm{p}}+\lambda\right) S-\delta S=0 \\ \delta S-\mu S_{M}-(1-\epsilon) \lambda S_{M}=0\end{array}\right.$

Solving the above equations with $\lambda=0$; (6) becomes

$S=\frac{\Lambda_{1}}{\left(\mu_{\mathrm{p}}+\lambda+\delta\right)}, \quad S^{0}=\frac{\Lambda_{1}}{\left(\mu_{\mathrm{p}}+\delta\right)}, S_{M,}^{0}=\frac{\Lambda_{1} \delta}{\left(\mu_{\mathrm{p}}+\delta\right)\left[\mu_{S_{M}}\right]}$

Thus the disease free equilibrium point is stated as:

$E^{0}=\left(S^{0}, I^{0}, R^{0}, S_{M}^{0}, B^{0}\right)\left(\frac{\Lambda_{1}}{\left(\mu_{\mathrm{p}}+\delta\right)}, 0,0, \frac{\Lambda_{1} \delta}{\left(\mu_{\mathrm{p}}+\delta\right)\left[\mu_{S_{M}}\right]}, 0,\right)$

In the absence of infection the system will consist of two compartment classes the susceptible and susceptible with media information.

We calculate the basic reproduction number using the next generation operator method by [30]. We use $F$ to show matrix of new infections and $V$ the matrix of transfer of infection.

$\left\{\begin{array}{c}F=\left[\begin{array}{c}\lambda_{1} S+(1-\epsilon) \lambda_{1} S_{M} \\ \Lambda_{2}+(1-\epsilon) \theta I\end{array}\right] \\ V=\left[\begin{array}{c}\left(\alpha+\mu_{I}+\sigma\right) I \\ \left(\mu_{S}+\omega\right) B\end{array}\right]\end{array}\right.$

The product of $\mathrm{FV}^{-1}$ is given as where $V^{-1}$ is the inverse of $V$ :

$F V^{-1}=\left[\begin{array}{cc}0 & \frac{\beta \Lambda_{1}\left(\mu_{S_{M}}+(1-\epsilon) \delta\right)}{\left(\mu_{p}+\delta\right)\left(\mu_{S}+\omega\right) \mu_{S_{M}}} \\ \frac{(1-\epsilon) \theta}{\left(\alpha+\mu_{I}+\sigma\right)} & 0\end{array}\right]$

The basic reproduction number takes the largest Eigen value of $\mathrm{FV}^{-1}$ :

$$
\mathrm{R}_{0}=\frac{\sqrt{\beta} \sqrt{-1+\epsilon} \sqrt{\theta} \sqrt{\Lambda_{1}}}{\sqrt{\alpha+\sigma+\mu_{i}} \sqrt{\delta+\mu_{p}} \sqrt{\omega+\mu_{S}} \sqrt{\mu_{S_{M}}}}
$$

\section{E. Local Stability of Disease Free Equilibrium Point}

1) Theorem 1

A DFE point is locally asymptomatically stable if $R_{0}<1$, otherwise unstable.

Proof: We compute the Eigen values of the Jacobian matrix of the model (1). The Jacobian- matrix is obtained by differentiating each of the equations in the system with respect to $S^{0}, I^{0}, R^{0}, S_{M}{ }^{0}, B^{0} . \lambda=\beta B^{0}, B^{0}=0$ at DFE $\boldsymbol{E}^{\mathbf{0}}$. 
$J_{E_{0}}=\left[\begin{array}{ccccc}-\left(\mu_{\mathrm{p}}+\delta\right) & 0 & \tau & 0 & 0 \\ 0 & -\left(\alpha+\mu_{I}+\sigma\right) & 0 & 0 & 0 \\ 0 & \alpha & -\left(\mu_{R}+\tau\right) & 0 & 0 \\ \delta & 0 & 0 & -\mu_{S_{M}} & 0 \\ 0 & (1-\epsilon) \theta & 0 & 0 & -\left(\mu_{s}+\omega\right)\end{array}\right]$

The Eigen values of the above matrix are $\left\{-\alpha-\mu_{I}-\sigma,-\mu_{\mathrm{P}}-\delta,-\mu_{R}-\tau,-\mu_{S}-\omega,-\mu_{S_{M}}\right.$,

It is evident that all the Eigen values are negative thus the disease free equilibrium point is locally asymptomatically stable.

\section{F. Global Stability of Disease Free Equilibrium}

The criteria that is used to determine the condition necessary for global asymptotic stability of the DFE is the Lyapunov criterion for stability. To prove the global stability, we state and prove the following theorem.

\section{1) Theorem and Proof}

The DFE equilibrium model is globally asymptomatically stable if $R_{0}{ }^{*}<1$ and unstable if $R_{0}{ }^{*}>1$. We consider a linear and logarithmic Lyapunov function for the system of (1) as follows:

$G\left(S, I, R, S_{m}, B\right)=S-S^{O}-S^{0} \ln \left(\frac{S}{S^{0}}\right)+k_{1} I+k_{2} R+k_{3} S_{m}+k_{4} B$

A Lyapunov function $\mathrm{G}$ satisfies the following conditions to guarantee global asymptomatic stability:

i) $G\left(S^{O}, I^{O}, R^{O}, S_{m}{ }^{O}, B^{0}\right)=0$ and $G\left(S, I, R, S_{m}, B\right)>0$ for $S, I, R, S_{m}, B \neq 0$

ii) $\frac{d\left(S^{O}, I^{O}, R^{O}, S_{m}{ }^{O}, B^{0}\right)}{d t}=0$ and $\frac{d\left(S, I, R, S_{m}, B\right)}{d t}<0$.

iii)

At DFE, $G\left(S^{O}, I^{O}, R^{O}, S_{m}{ }^{o}, B^{0}\right)=S^{0}-S^{O}-S^{0} \ln \left(\frac{S^{O}}{S^{0}}\right)+k_{1} I^{0}+k_{2} R^{O}+k_{3} S_{m}{ }^{o}+k_{4} B^{O}$

Since $I^{O}=R^{O}=S_{m}{ }^{O}=B^{0}=0$ and $\ln 1=0 G\left(S^{O}, I^{O}, R^{O}, S_{m}{ }^{O}, B^{0}\right)=0$ Condition (I) is satisfied. In the same sense, $S \neq$ $S^{O}$ and $I \neq 0, R \neq 0, S_{m} \neq 0, B \neq 0$ and $\ln \left(\frac{S}{S^{0}}\right) \neq 0$, thus $G\left(S, I, R, S_{m}, B\right)>0$

Condition (ii) $\frac{d\left(S, I, R, S_{m}, B\right)}{d t}=\frac{d S}{d t}\left(1-\frac{S^{0}}{S}\right)+k_{1} \frac{d I}{d t}+k_{2} \frac{d R}{d t}+k_{3} \frac{d S_{m}}{d t}+k_{4} \frac{d B}{d t}$

At DFE $\frac{d\left(s^{O}, I^{O}, R^{O}, S_{m}{ }^{o}, B^{0}\right)}{d t}=0$ Since $I^{O}=R^{O}=S_{m}{ }^{O}=B^{0}=0,\left(1-\frac{s^{0}}{S^{o}}\right)=0$ hence the condition $\frac{d\left(S, I, R, S_{m}, B\right)}{d t}<0$ will help us to ascertain the Global stability of DFE

Substituting for $\frac{d S}{d t}, \frac{d I}{d t}, \frac{d R}{d t}, \frac{d S_{m}}{d t}, \frac{d B}{d t}$ we have

$$
\begin{gathered}
\frac{d\left(S, I, R, S_{m}, B\right)}{d t}=\left(1-\frac{s^{0}}{S}\right)\left(\Lambda_{1}+\tau R-\left(\mu_{p}+\lambda\right) S-\delta S\right)+k_{1}\left(\lambda S+(1-\epsilon) \lambda S_{m}-\left(\alpha+\mu_{I}+\sigma\right) I\right)+k_{2}\left(\alpha I-\left(\mu_{R}+\tau\right) R\right)+ \\
k_{3}\left(\delta S-\mu_{s m} S_{m}-(1-\epsilon) \lambda S_{m}\right)+k_{4}\left(\Lambda_{2} B+(1-\epsilon) \theta I-\left(\mu_{s}+\omega\right) B\right)
\end{gathered}
$$

By grouping linear and non-linear terms we obtain the values of $k_{1}, k_{2}, k_{3}, k_{4}$ and and substituting $k_{1}=1, k_{2}=1, k_{3}=$ 1 and $k_{4}=0$ into the equation (14) and expanding we obtain and putting the positives and negatives together we obtain:

$\frac{d\left(S, I, R, S_{m}, B\right)}{d t}=\Lambda_{1}+\tau R+\mu_{p} S^{0}+\lambda S^{0}+\delta S^{0}+\lambda S+\lambda S_{m}+\delta S+\epsilon \lambda S_{m}-\mu_{p} S-\lambda S-\delta S-\Lambda_{1} \frac{S^{0}}{S}-\tau R \frac{S^{0}}{S}-\epsilon \lambda S_{m}-\alpha I-$ $\mu_{I} I-\sigma I+\alpha I-\mu_{R} R-\tau R-\mu_{s m} S_{m}-\lambda S_{m}$

Letting $\mathrm{P}$ and $\mathrm{Q}$ be positive and negative terms respectively then we have, $\frac{d\left(S, I, R, S_{m}, B\right)}{d t}=P-Q$ (17). If $Q>P$ then the system is globally asymptotically stable otherwise unstable.

\section{G. Endemic Equilibrium Point}

Endemic equilibrium exists whenever $R_{0}>1$ and it is asymptomatically stable. As $R_{0}$ increases through one there is an exchange of stability between disease free equilibrium and endemic equilibrium point. Endemic equilibrium point is obtained by setting the right hand side of the model equations 1 to zero and that, $B>0, I>0, \lambda>0, \mathrm{~S}>0, \mathrm{R}>0$. We solve model of equations 1 in terms of force of infection $\lambda=\beta B . \lambda^{*}=\lambda$. We obtain $S^{*}, I^{*}, R^{*}, S_{M}{ }^{*}, B^{*}$. By letting $\mu_{p}=\mu_{1}, \mu_{I}=\mu_{4,} \mu_{S_{M}}=$ $\mu_{2,}, \mu_{R}=\mu_{3}, \mu_{s}=\mu_{5}$. 


$$
\left\{\begin{array}{c}
S^{*}=\frac{-\alpha \tau \mu_{2}+\Lambda_{1}\left(\tau+\mu_{3}\right)\left(\alpha+\sigma+\mu_{4}\right)}{(\delta \sigma-\lambda(2 \alpha+\sigma)) \tau+\mu_{1}\left(\tau+\mu_{3}\right)\left(\alpha+\sigma+\mu_{4}\right)+(\delta-\lambda)\left(\tau \mu_{4}+\mu_{3}\left(\alpha+\sigma+\mu_{4}\right)\right)} \\
I^{*}=\frac{\left((\delta+\lambda) \Lambda_{1}-\left(\delta-\lambda+\mu_{1}\right) \mu_{2}\right)\left(\tau+\mu_{3}\right)}{(\delta \sigma-\lambda(2 \alpha+\sigma)) \tau+\mu_{1}\left(\tau+\mu_{3}\right)\left(\alpha+\sigma+\mu_{4}\right)+(\delta-\lambda)\left(\tau \mu_{4}+\mu_{3}\left(\alpha+\sigma+\mu_{4}\right)\right)} \\
\mathrm{R}^{*}=\frac{\alpha(\delta+\lambda) \Lambda_{1}-\alpha\left(\delta-\lambda+\mu_{1}\right) \mu_{2}}{(\delta \sigma-\lambda(2 \alpha+\sigma)) \tau+\mu_{1}\left(\tau+\mu_{3}\right)\left(\alpha+\sigma+\mu_{4}\right)+(\delta-\lambda)\left(\tau \mu_{4}+\mu_{3}\left(\alpha+\sigma+\mu_{4}\right)\right)} \\
S_{M}{ }^{*}=\frac{\left(\delta \Lambda_{1}\left(\tau+\mu_{3}\right)\left(\alpha+\sigma+\mu_{4}\right)-\mu_{2}\left((\alpha(\delta-2 \lambda)+(\delta-\lambda) \sigma) \tau+\mu_{1}\left(\tau+\mu_{3}\right)\left(\alpha+\sigma+\mu_{4}\right)+(\delta-\lambda)\left(\tau \mu_{4}+\mu_{3}\left(\alpha+\sigma+\mu_{4}\right)\right)\right)\right)}{\left((-1+\epsilon) \lambda\left((2 \alpha \lambda+(-\delta+\lambda) \sigma) \tau-\mu_{1}\left(\tau+\mu_{3}\right)\left(\alpha+\sigma+\mu_{4}\right)-(\delta-\lambda)\left(\tau \mu_{4}+\mu_{3}\left(\alpha+\sigma+\mu_{4}\right)\right)\right)\right)} \\
B^{*}=\frac{(-1+\epsilon) \theta \Lambda_{2}\left((\delta+\lambda) \Lambda_{1}-\left(\delta-\lambda+\mu_{1}\right) \mu_{2}\right)\left(\tau+\mu_{3}\right)}{\left((2 \alpha \lambda+(-\delta+\lambda) \sigma) \tau-\mu_{1}\left(\tau+\mu_{3}\right)\left(\alpha+\sigma+\mu_{4}\right)-(\delta-\lambda)\left(\tau \mu_{4}+\mu_{3}\left(\alpha+\sigma+\mu_{4}\right)\right)\right)\left(\omega+\mu_{5}\right)}
\end{array}\right.
$$

Using equations 18 we substitute them in the force of infection to obtain $\lambda^{*}$ the force of infection at endemic equilibrium point in terms of $\beta$. We obtain two values of $\lambda^{*}$ as:

$\lambda^{*}{ }_{1}=\rho_{1}-2 \frac{R_{0}^{2}}{\Lambda_{1}} \rho_{2}+\rho_{4}+\rho_{3}-R_{0}^{2} \rho_{2}$

$\lambda^{*}{ }_{2}=\rho_{1}-2 \frac{R_{0}^{2}}{\Lambda_{1}} \rho_{2}+\rho_{4}-\rho_{3}-R_{0}^{2} \rho_{2}$

Substituting $\beta^{*}$ in equations with $\lambda^{*}{ }_{1}$ and then substituting $\lambda^{*}{ }_{1}$ in (18) we obtain $S^{*}, I^{*}, R^{*}, S_{M}{ }^{*}, B^{*}$ in terms of $R_{0}$. Let $\lambda^{*}{ }_{1}=\lambda$ and $\lambda^{*}{ }_{1}=\xi R_{0}$.

$$
\left\{\begin{array}{c}
S^{*}=\left[\frac{-\alpha \tau \mu_{2}+\left(\alpha+\sigma+\mu_{4}\right) \Lambda_{1}\left[\tau+\mu_{3}\right]}{\left(\alpha+\sigma+\mu_{4}\right) \mu_{1}\left[\tau+\mu_{3}\right]+\left(\delta-\xi \mathrm{R}_{0}\right)\left(\tau \mu_{4}+\mu_{3}\left[\alpha+\sigma+\mu_{4}\right]\right)+\tau\left(\delta \sigma-\xi \mathrm{R}_{0}[2 \alpha+\sigma]\right)}\right] \\
I^{*}=\left[\frac{\left(\tau+\mu_{3}\right)\left(-\mu_{2}\left(\delta+\mu_{1}-\xi \mathrm{R}_{0}\right)+\Lambda_{1}\left(\delta+\xi \mathrm{R}_{0}\right)\right)}{\left(\alpha+\sigma+\mu_{4}\right) \mu_{1}\left[\tau+\mu_{3}\right]+\left(\delta-\xi \mathrm{R}_{0}\right)\left(\tau \mu_{4}+\mu_{3}\left[\alpha+\sigma+\mu_{4}\right]\right)+\tau\left(\delta \sigma-\xi \mathrm{R}_{0}[2 \alpha+\sigma]\right)}\right] \\
\mathrm{R}^{*}=\left[\frac{-\mu_{2} \alpha\left[\delta+\mu_{1}-\xi \mathrm{R}_{0}\right]+\Lambda_{1} \alpha\left[\delta+\xi \mathrm{R}_{0}\right]}{\left(\alpha+\sigma+\mu_{4}\right) \mu_{1}\left[\tau+\mu_{3}\right]+\left(\delta-\xi \mathrm{R}_{0}\right)\left(\tau \mu_{4}+\mu_{3}\left[\alpha+\sigma+\mu_{4}\right]\right)+\tau\left(\delta \sigma-\xi \mathrm{R}_{0}[2 \alpha+\sigma]\right)}\right]
\end{array}\right]
$$

Thus, the endemic equilibrium point is stated as $E^{*}$ and exists if $R_{0}>1$.

$$
E^{*}=\left(S^{*}, I^{*}, R^{*}, S_{M}{ }^{*}, B^{*}\right)
$$

\section{H. Bifurcation of the Model}

Bifurcation analysis is done using Centre Manifold theory [17]. To apply centre Manifold theory the change of variables is done first for simplicity. Let $S=x_{1}, I=x_{2}, R=x_{3}, S_{M}=x_{4}, B=x_{5}$ then $N=x_{1}+x_{2}+x_{3},+x_{4}+x_{5}$.

The model can be written as $\frac{d N}{d x}=F(x)$ where $=\left(f_{1}, f_{2}, f_{3}, f_{4}, f_{5}\right) \lambda^{*}=\beta^{*} B$, where $\beta^{*}$ is the bifurcation parameter.

$$
\left\{\begin{array}{c}
f_{1}=\frac{d x_{1}}{d t}=\Lambda_{1}+\tau \mathrm{R}-\left(\mu_{\mathrm{p}}+\beta^{*} x_{5}\right) \mathrm{x}_{1}-\delta \mathrm{x}_{1} \\
f_{2}=\frac{d x_{2}}{d t}=\beta^{*} x_{5} x_{1}+(1-\epsilon) \beta^{*} x_{5} \mathrm{x}_{4}-\left(\alpha+\mu_{I}+\sigma\right) x_{2} \\
f_{3}=\frac{d x_{3}}{d t}=\alpha x_{2}-\left(\mu_{R}+\tau\right) x_{3} \\
f_{4}=\frac{d x_{4}}{d t}=\delta x_{1}-\mu_{S_{M}} x_{4}-(1-\epsilon) \beta^{*} x_{5} \mathrm{x}_{4} \\
f_{5}=\frac{d x_{5}}{d t}=\Lambda_{2}+(1-\epsilon) \theta x_{2}-\left(\mu_{S}+\omega\right) x_{5}
\end{array}\right.
$$

The method entails obtaining the Jacobian of the system of (22) at disease free equilibrium $E^{0}=\left(S^{0}, I^{0}, R^{0}, S_{M}^{0}, B^{0}\right)=$ $\left(\frac{\Lambda_{1}}{\left(\mu_{p}+\delta\right)}, 0,0, \frac{\Lambda_{1} \delta}{\left(\mu_{p}+\delta\right)\left[\mu_{S_{M}}\right]}, 0,\right)$.

We consider a case where $R_{0}=1$, we take $\beta=\beta^{*}$ as the bifurcation parameter. Then from $R_{0}{ }^{*}=1$ gives $\beta^{*}=$ $\left.\left.-\frac{\left(\alpha+\sigma+\mu_{i}\right)\left(\delta+\mu_{p}\right)\left(\omega+\mu_{S}\right) \mu_{S_{M}}}{(-1+\epsilon) \theta \Lambda_{1} \Lambda_{2}\left(\delta-\delta \epsilon+\mu_{S_{M}}\right)}\right\}\right\}$ the Jacobean of $\frac{d N}{d x}=F(x)$ at disease free equilibrium when $\beta=\beta^{*}$ denoted by $J E_{*}^{0}$ with $\beta=\beta^{*}$ has the Eigen values: 


$$
J E_{*}^{0}=J \beta^{*}=\left[\begin{array}{ccccc}
-\mu_{\mathrm{p}}-\delta & 0 & 0 & 0 & -\beta^{*} \frac{\Lambda_{1} \delta}{\left(\mu_{\mathrm{p}}+\delta\right)\left[\mu_{S_{M}}\right]} \\
0 & -\left(\alpha+\mu_{I}+\sigma\right) & 0 & 0 & 0 \\
0 & \alpha & -\left(\mu_{R}+\tau\right) & 0 & 0 \\
\delta & 0 & 0 & -\mu_{S_{M}} & -\beta^{*} \frac{\Lambda_{1} \delta}{\left(\mu_{\mathrm{p}}+\delta\right)\left[\mu_{\left.S_{M}\right]}\right]} \\
0 & (1-\epsilon) \theta & 0 & 0 & \left(\mu_{S}+\omega\right)
\end{array}\right]
$$

Obtaining the Eigen values the above Jacobean matrix at DFE and obtaining $\beta=\beta^{*}$ interms of $R_{0}$ such that $R_{0} \beta^{*}=1$. We obtain the Eigen values as:

$$
\left\{0,-\delta+-\mu_{p},-\alpha-\sigma-\mu_{i},-\tau-\mu_{R}, \omega+\mu_{S}\right\}
$$

It can be shown that the Jacobian of (22) has five Eigen values with zero being one of them. The centre manifold theory used by [17] is used to analyze the dynamics of the model. The theorem stated below was used to analyze the dynamics of the model by [24].

Taking $\beta^{*}$ as the bifurcation parameter such that:

$\beta^{*}=-\frac{\mathrm{R}_{0}^{2}\left(\alpha+\sigma+\mu_{\mathrm{i}}\right)\left(\delta+\mu_{\mathrm{p}}\right)\left(\omega+\mu_{\mathrm{S}}\right) \mu_{\mathrm{S}_{\mathrm{M}}}}{(-1+\epsilon) \theta \Lambda_{1} \Lambda_{2}\left(\delta-\delta \epsilon+\mu_{S_{M}}\right)}$ When $\mathrm{R}_{0}=1 \beta^{*}=-\frac{\left(\alpha+\sigma+\mu_{\mathrm{i}}\right)\left(\delta+\mu_{\mathrm{p}}\right)\left(\omega+\mu_{\mathrm{S}}\right) \mu_{\mathrm{S}_{\mathrm{M}}}}{(-1+\epsilon) \theta \Lambda_{1} \Lambda_{2}\left(\delta-\delta \epsilon+\mu_{\mathrm{S}_{\mathrm{M}}}\right)}$

It follows that when $\beta=\beta^{*}$ the DFE satisfies the theorem that zero is a simple Eigen value and all other Eigen values have negative real parts. If $\beta^{*}$ is taken as the bifurcation parameter, we have a right Eigen vector $u=\left(\begin{array}{lllll}u_{1} & u_{2} & u_{3} & u_{4} & u_{5}\end{array}\right)^{T}$ associated with the zero Eigen value and a left Eigen vector $v=\left(v_{1}, v_{2}, v_{3}, v_{4}, v_{5}\right)^{T}$ associated with the zero Eigen value satisfying the condition $\mathrm{u} . \mathrm{v}=1$.

\section{Eigen Vectors}

$J E_{*}^{0}=J \beta^{*}$ The Jacobean of the model $J \beta^{*}$ has a right Eigen vector denoted by $u=\left(\begin{array}{lllll}u_{1} & u_{2} & u_{3} & u_{4} & u_{5}\end{array}\right)^{T}$.

$$
\left[\begin{array}{ccccc}
-\mu_{\mathrm{p}}-\delta & 0 & 0 & 0 & -\beta^{*} \frac{\Lambda_{1} \delta}{\left(\mu_{\mathrm{p}}+\delta\right)\left[\mu_{S_{M}}\right]} \\
0 & -\left(\alpha+\mu_{I}+\sigma\right) & 0 & 0 & 0 \\
0 & \alpha & -\left(\mu_{R}+\tau\right) & 0 & 0 \\
\delta & 0 & 0 & -\mu_{S_{M}} & -\beta^{*} \frac{\Lambda_{1} \delta}{\left(\mu_{\mathrm{p}}+\delta\right)\left[\mu_{S_{M}}\right]} \\
0 & (1-\epsilon) \theta & 0 & 0 & -\left(\mu_{S}+\omega\right)
\end{array}\right]\left[\begin{array}{l}
u_{1} \\
u_{2} \\
u_{3} \\
u_{4} \\
u_{5}
\end{array}\right]=\left[\begin{array}{l}
0 \\
0 \\
0 \\
0 \\
0
\end{array}\right]
$$

$$
\text { Let }-\left(\mu_{\mathrm{p}}+\delta\right)=-\mathrm{k}_{1},-\left(\alpha+\mu_{I}+\sigma\right)=-\mathrm{k}_{2},-\left(\mu_{R}+\tau\right)=-\mathrm{k}_{3}, \Lambda_{2}-\left(\mu_{S}+\omega\right)=-\mathrm{k}_{4}, \frac{\Lambda_{1} \delta}{\left[\mu_{S_{M}}\right]}=\mathrm{k}_{5},(1-\epsilon)=\mathrm{k}_{6} \text {. }
$$

solving we obtain $\left\{\begin{array}{c}\boldsymbol{u}_{1}=\frac{-\beta^{*} \mathrm{k}_{5} u_{5}}{k_{1}{ }^{2}}<0 \\ \boldsymbol{u}_{2}=0 \\ \boldsymbol{u}_{3}=0 \\ {\boldsymbol{\beta _ { 4 }}}_{4}=\frac{\frac{\beta^{*} \mathrm{k}_{5}}{k_{1} u_{5}+\delta u_{1}}}{\mu_{S_{M}}}>0 \\ \boldsymbol{u}_{5}=\frac{u_{1} k_{1}^{2}}{-\beta^{*} \mathrm{k}_{5}}>0\end{array}\right.$

The Jacobean has a left Eigen vector given $v$ denoted by $v=\left(v_{1}, v_{2}, v_{3}, v_{4}, v_{5}\right)^{T}$ which satisfies the condition $u . v=1$. Solving we obtain,

$$
\left\{\begin{array}{c}
v_{1}=\frac{\delta v_{4}}{k_{1}}>0 \\
v_{2}=\frac{k_{6} \theta v_{5}}{k_{2}}>0 \\
v_{3}=0 \\
v_{4}=0 \\
v_{5}=0
\end{array}\right.
$$


We calculate the value of $\boldsymbol{a}$ using $v_{k} u_{i} u_{j} \frac{\partial^{2} f_{k}}{\partial x_{i} \partial x_{k}}$ Calculating the partial derivatives at disease free equilibrium by taking $k=$ $1,2, ; \mathrm{i}, \mathrm{j}=1,4,5$. and adding the non-zero partial derivatives we obtain the value of $\boldsymbol{a}, \boldsymbol{a}=\boldsymbol{v}_{2} \mathrm{u}_{1} \mathrm{u}_{5}\left(\beta+\mathrm{u}_{4}(1-\epsilon) \beta+\boldsymbol{v}_{1} \beta\right)<$ $0, a<0$ Because $u_{1}<0, v_{2}, u_{4}, u_{5}>0$. To obtain the value of $\mathbf{b}$ we use $\mathrm{b}=\sum_{i j=1}^{n} v_{k} u_{i} \frac{\partial^{2} f_{k}}{\partial x_{i} \partial \boldsymbol{\beta}^{*}}$, we calculate the partial derivatives with respect to $\boldsymbol{\beta}^{*}$ and by taking $k=1,2 ; \mathrm{i}, \mathrm{j}=1,4,5$. Adding the non zero partial derivatives with respect to $\boldsymbol{\beta}^{*}$ we obtain the value of $\mathbf{b}$.

$\mathrm{b}=v_{2} u_{5}(1-\epsilon) \frac{\Lambda_{1} \delta}{\left(\mu_{\mathrm{p}}+\delta\right)\left[\mu_{S_{M}}\right]}+v_{1} u_{5} \frac{\Lambda_{1}}{\left(\mu_{\mathrm{p}}+\delta\right)}>0$. From theorem 5 (III); $a<0$ and $b>0$ when. Backward bifurcation does not occur hence it shows that it is possible to control bilharzia.

\section{J. Sensitivity Analysis}

The expression of $R_{0}$ is given in equation (10). Our $R_{0}$ depends on mass media campaign therefore we derive our sensitivity to each parameter using the normalized forward sensitivity index by [7]. The parameter values used in simulation and sensitivity index are; $\Lambda_{2}=0.76, \Lambda_{1}=0.015, \beta=0.0002473419203324199, \theta=0.36, \alpha=0.7000, \sigma=0.0586, \mu_{I}=$ $0.003000, \delta=0.6579, \mu_{p}=0.00500, \mu_{S_{M}=}, 0.00250, \epsilon=0.3987, \omega=0.063, \mu_{S}=0.15, \tau=0.1083, \mu_{R}=0.005464$.

TABLE I: PARAMETERS AND THEIR SENSITIVITY INDICES

\begin{tabular}{cc}
\hline \hline Parameter & Sensitivity Index \\
\hline \hline$\Lambda_{2}$ & +0.999863 \\
$\Lambda_{1}$ & +0.00006854 \\
$\beta$ & +0.00006854 \\
$\theta$ & +0.00006854 \\
$\alpha$ & -0.0000453261 \\
$\sigma$ & -0.00000379444 \\
$\mu_{I}$ & -0.000019425 \\
$\delta$ & +0.00000233213 \\
$\mu_{p}$ & -0.000048415 \\
$\mu_{S_{M}}$ & -0.0000660367 \\
$\epsilon$ & -0.0000454504 \\
$\omega$ & -0.294626 \\
$\mu_{S}$ & -0.703077 \\
$\tau$ & 0.000 \\
$\mu_{R}$ & 0.000 \\
\hline \hline
\end{tabular}

\section{K. Numerical Simulation}

Numerical simulations of the model system (1) are carried out using a set of parameter values given in Table II. Matlab $\mathrm{R} 2015 \mathrm{a}$ is used in the numerical simulation to obtain results. Secondary (primary) data collected from Mwea irrigation scheme by [25] was used to estimate human populations and parameters used in the model while secondary data by [9] was used to estimate the snail population and other parameters relating to the snails. Graphical representations showing variations in different populations with respect to a given period of time $(t)$ after exposure to media campaigns are given in Fig. 2-6. Most parameter values were obtained from literature and those not available from literature were estimated.

TABLE II: PARAMETERS VALUES USED IN SIMULATIONS

\begin{tabular}{ccc}
\hline \hline $\begin{array}{c}\text { Parameter } \\
\text { symbol }\end{array}$ & Value out of 100 & Source \\
\hline \hline$\Lambda_{1}$ & 0.015 & Okonjo Edward et al \\
$\mathrm{S}$ & 325142 & Okonjo Edward et al \\
$\mathrm{S}_{\mathrm{M}}$ & 129634 & Okonjo Edward et al \\
$I$ & 75108 & Okonjo Edward et al \\
$R$ & 49413 & Okonjo Edward et al \\
$\Lambda_{2}$ & 0.76 & D.M. Ngigi et al \\
$\boldsymbol{\delta}$ & 0.6579 & Okonjo Edward et al \\
$\mu$ & 0.00500 & KNBS,KPHC 2019 \\
$\sigma$ & 0.0586 & Okonjo Edward et al \\
$\lambda$ & 0.6785 & Okonjo Edward et al \\
$\alpha$ & 0.7000 & Okonjo Edward et al \\
$\epsilon$ & 0.3987 & Okonjo Edward et al \\
$\tau$ & 0.1083 & Okonjo Edward et al \\
$\theta$ & 0.36 & D.M. Ngigi et al \\
$\mu_{s}$ & 0.15 & D.M. Ngigi et al \\
$\omega$ & 0.063 & D.M. Ngigi et al \\
$\mathrm{B}$ & 905 & D.M. Ngigi et al \\
$\mu_{I}$ & 0.003000 & Estimated \\
$\mu_{R}$ & 0.005464 & Estimated \\
$\mu_{s m}$ & 0.00250 & Estimated \\
\hline \hline
\end{tabular}




\section{1) Graphical Presentation of Results}

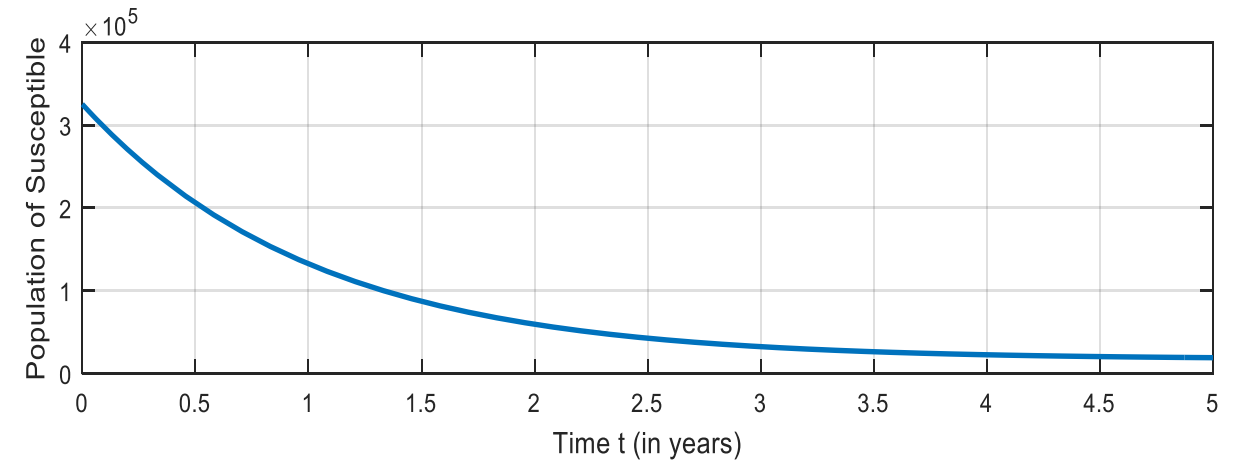

Fig. 2. Variation of populations of susceptible with respect to time.

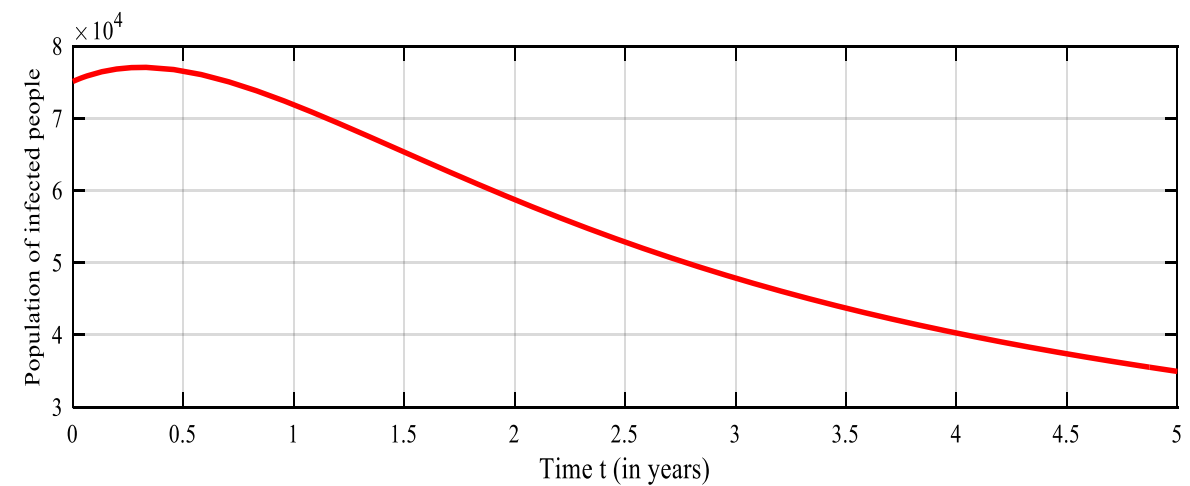

Fig. 3. Variations of populations of infected people against time

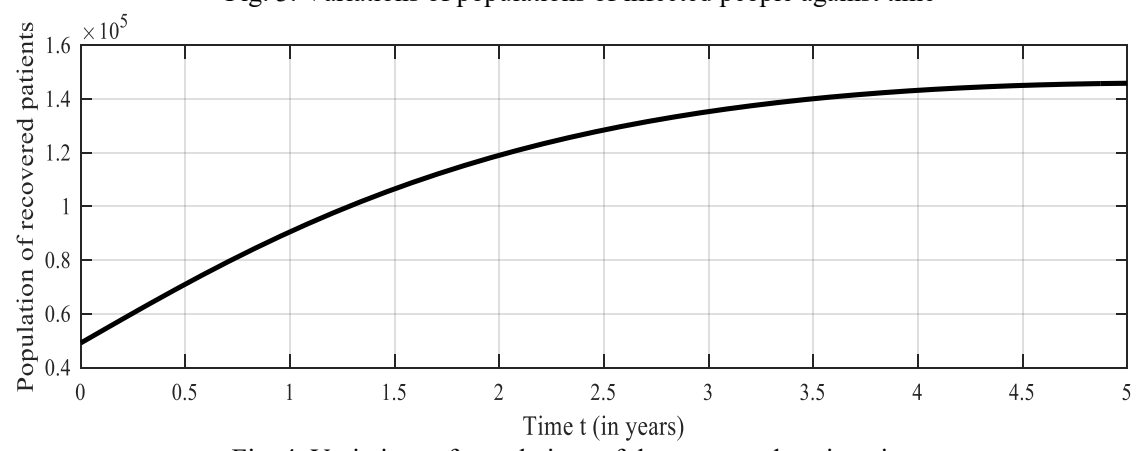

Fig. 4. Variations of populations of the recovered against time.

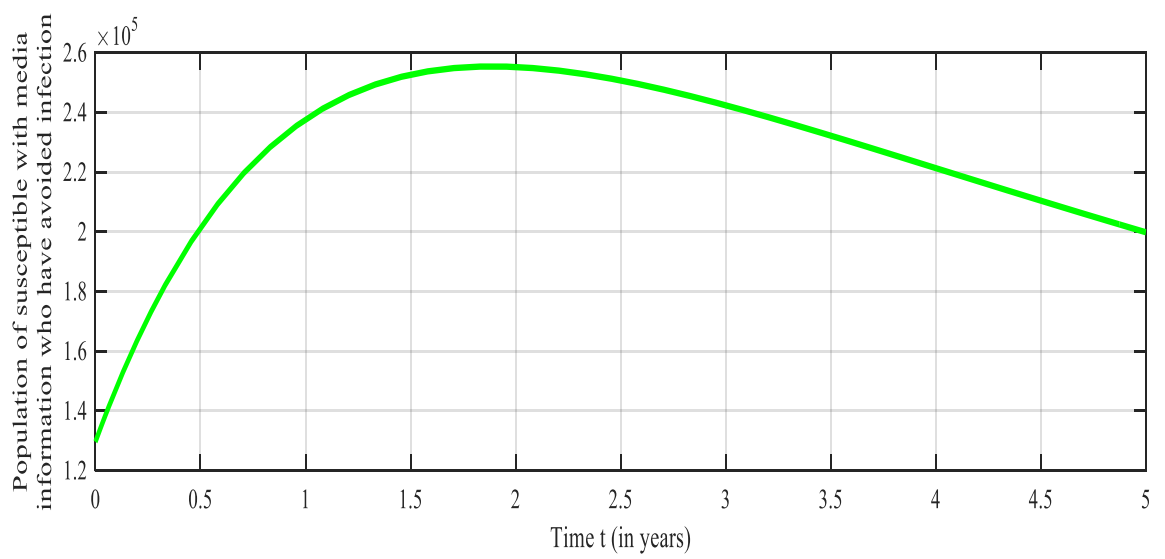

Fig. 5. Variation of populations of the susceptible with media information who have avoided infection against time 


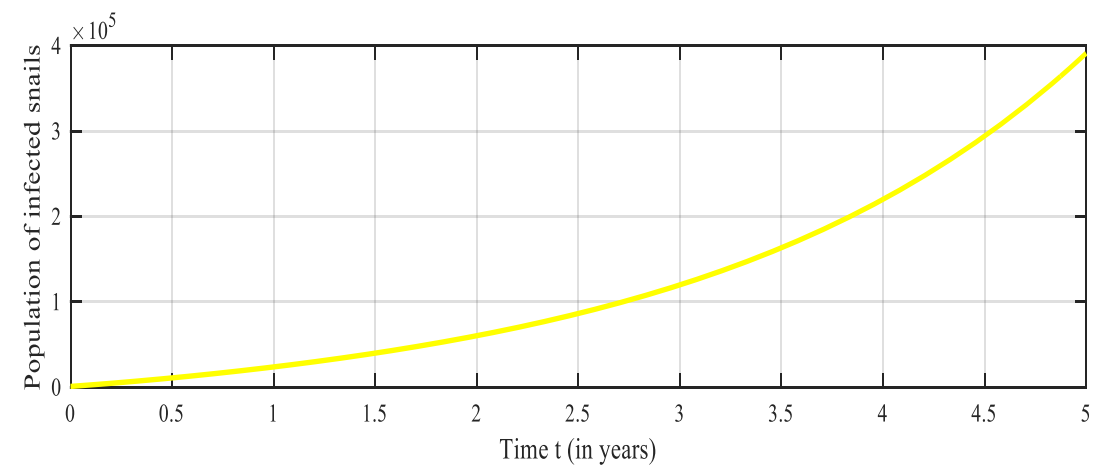

Fig. 6. Variations of populations of infected snails against time.

\section{DisCUSSION AND CONCLUSION}

From the graphical results it is observed that when media campaigns are applied as a control strategy cause a reduction of transmission of bilharzia in Mwea irrigation scheme. From our findings it is observed that when media campaigns are done for a period of five years there is a reduction in the number of the susceptible, infectious and an increase in the number of recoveries and those who get information and avoid infections. It also noted that the snail populations will increase without increasing the infections because awareness will make people avoid direct contact with infected environment. Therefore there is a reasonable agreement between the results of our model and those done by other authors who have suggested that there is need for a more integrated approach putting emphasis on provision of health education and awareness to be considered as a control strategy of the disease in Mwea irrigation scheme and other endemic areas, since drug administration alone was not adequate in alleviating the disease burden. This proves the fidelity of our equations and the numerical scheme used to solve these equations.

\section{REFERENCES}

[1] Abiola, F. A. (2015). Impact of human schistosomiasis in sub-Saharan Africa. Brazilian journal of infectious diseases, 19(2):196-205.

[2] Ahmad J., A. K. (2017). Factors associated with Schistosomiasis control measures in Mwaluphamba Location, Kwale County. Kenya Healthcare in Lowresource Settings, 5:6108.

[3] Akinyi, O. C. (2013). Modelling the Impact of Misdiagnosis and Treatment on the Dynamics of Malaria Concurrent and Co-infection with Pneumonia. Applied Mathematical Sciences Vol. 7 no. 126, 6275 - 6296.

[4] Chan M. S and Bundy, D. (1997). modeling the dynamic effects of community chemotherapy, on patterns of morbidity due to schistosoma mansoni. Transactions of the Royal Society of Tropical Medicine and Hygiene, , 91(2): 216-220 https://doi.org/10.1016/S0035 9203(97)90231-5.

[5] Chan, M. A. (1996). Dynamic aspects of morbidity and acquired immunity in schistosomiasis control. . Acta Trop, 62: 105-117.

[6] Charles, H. K. (2010). Parasites and poverty: The case of schistosomiasis: . Acta Tropica 113, 95-104.

[7] Chitnis, N. H. (2008). .Determining important Parameters in the spread of malaria through the sensitivity analysis of a mathematical Model. Bulletin of Mathematical Biology 70 (5), 1272-12.

[8] D, B. A. ((1994). Modeling the transmission of schistosomiasis;. an introductory view Winterthurertrasse, 190:8057.

[9] D. M. Ngigi, S. C. (2019). The Susceptibility of Biomphalaria spp. from Mwea Irrigation Scheme in Kenya against Schistosoma mansoni Miracidia Infection. Journal of biomedical science and engineering vol12,(No 11, 477-486.

[10] Diekman, O. H. (1990). On the definition and Computation of the basic reproduction ratio in the model of infectious disease in Heterogeneous populations. Journal of Mathematical Biology. 2(1), 265-382.

[11] Evans Asena Chadeka, S. N. (2019). A high-intensity cluster of Schistosoma mansoni infection around Mbita causeway, western Kenya: a confirmatory crosssectional survey. BMC Tropical Medicine and Health, 47:26.

[12] Fenga, X., \& c, Z. T. (2016.). Backward Bifurcation and Global Stability in an Epidemic Model with Treatment and Vaccination. Discrete and Continuous Dynamical Systems Series B doi:10.3934/dcdsb.2014.19.999, 999-1025.

[14] Fulford, A. B. (1992). On the use of age-intensity data to detect immunity to parasitic infections, with special reference to Schistosoma mansoni in Kenya. Parasitology, 105 (2, 219-227.

[15] Gurarie, D. (2015). Modeling control of Schistosoma haematobium infection: predictions of the long-term impact of mass drug administration in Africa. Parasites \& Vectors 8:529 DOI 10.1186/s13071-015-1144-3.

[16] JOHN H. OUMA, C. H. (2005). Late Benefits 10-18 Years after Drug Therapy for Infection with Schistosoma Haematobium in Kwale District, Coast Province, Kenya. Journal of Tropical Medicine. Hyg., 73(2), , , pp. 359-364.

[17] Liu, X. Z. (2011). backward bifurcation of an epdemic model with saturated treatment function. journal of mathematical analtical application, 433-443.

[18] Masaku J, M. N. (2015). Current status of Schistosoma mansoni and the factors associated with infection two years following mass drug administration programme among primary school children in Mwea irrigation scheme. BMC Public Health, 15:739. DOI: 10.1186/s12889-015-1991.

[19] Mutapi, F. M. (2017). Human schistosomiasis in the post Mass Drug Administration (MDA) era. . Lancet Infectious Diseases, 17(2):(doi:10.1016/S14733099(16)30475-3), e42-e48.

[20] Mutapi, F. N. (1998). Chemotherapy accelerates the development of acquired immune responses to Schistosoma haematobium infection. Infectious Diseases. 178, 289-298.

[21] Mwai J., N. S. (2016). Knowledge, attitude and practices in relation to prevention and control of schistosomiasis infection in Mwea Kirinyaga county, . Kenya BMC Public Health 16:819, DOI 10.1186/s12889-016-3494-y.

[22] Nadine Rujeni, N. N. (2012). Atopy is inversely related to schistosome infection intensity: a comparative study in Zimbabwean villages with distinct levels of Schistosoma haematobium infection. PubMed, PMC3398828 DOI: 10.1159/000332949.

[23] Nelwan, M. L. (2019). Schistosomiasisl Life cycle, Diagnosis and Control. current therapeutic research vol 19; https://doi.org/10.1016/j.curthers.2019.06.001, 5-9.

[24] Okaka, A. C. (2013). Modelling the Impact of Misdiagnosis and Treatment on the Dynamics of Malaria Concurrent and Co-infection with Pneumonia. Applied Mathematical Sciences Vol. 7 no. 126, 6275 - 6296.

[25] Okonjo Edward, ,. Y. (2017). Prevalence and Intensity of Schistosoma Mansoni before and after Chemotherapy in School Aged Children.,.. Journal of Natural Sciences Research ISSN 2224-3186 (Paper) ISSN 2225-0921, Vol.7, No.22, . 
[26] Paul M. Gichuki, S. K. (2019). Association between Schistosoma mansoni infection and access to improved water and sanitation facilities in Mwea,Kirinyaga County, Kenya. , BMC Infectious Diseases volume 19, Article number: 503 .

[27] Sammy M. Njenga, Y. I. (2019). gap between health education content and practice toward schistosomiasis prevention among schoolchildren along the shores of Lake Victoria in Kenya. PLOS Neglected Tropical Diseases | https://doi.org/10.1371/journal.pntd.0007572, 1-16.

[28] Shona, R. O. (2017). Interdependence between Schistosome Transmission and Protective Immunity. Tropical medicine and infectious diseases 2 ; doi:10.3390/tropicalmed2030042, 42 .

[29] van den Driessche P. (2017). Reproduction numbers of infectious disease models,. Infectious Disease Modelling (2017), doi: 10.1016/j.idm.2017.06.002., $1-30$

[30] Van den Driessche, P. a. (2002). Reproduction numbers and sub-threshold endemic equilibria for compartmental models of disease transmission. . Mathematical Biosciences, $180(1-2)$ :, 29-48.

[31] WHO. (2014, october 7th). Schistosomiasis: number of people treated in 2012. Wkly Epidemiol Rec. ;89(4). [PubMed] [Google Scholar]., pp. 21-28.

[32] WHO. (2017). Schistosomiasis. Available from: http://www.who.int/topics/.

[33] WHO. (2021). all children have a right to be protetected from preventable diseases. kenya: paeditric paziquintel consortium.

[34] WHO. (Updated January 2017). Schistosomiasis. http://www.who.int/mediacentre/factsheets/fs115/en/.: Media Centre.

[35] Woolhouse M E., E. J. (1998). Heterogeneities in schistosome transmission dynamics and control. Parasitology 117, 475-482.

[36] Woolhouse, M (1991). On the application of mathematical models of schistosome transmission dynamics I. Natural transmission. Acta Trop. 49:, 241270.

[37] World Health Organization, (2010). Weekly Epidemiological Record 18 (85):, 157-64. Http:/Www.Who.Int/Wer/En/.

[38] Yingke L., Z. T. (2017). A Mathematical Model for the Seasonal Transmission of Schistosomiasis in the Lake and Marshland Regions of China. Mathematical Biosciences and Engineering (14)(5\&6): Doi:10.3934/Mbe.2017066, 1279-1299. 